(1)

CrossMark

\title{
Are peripheral blood eosinophil counts a guideline for omalizumab treatment? STELLAIR says no!
}

\author{
William W. Busse
}

Affiliation: Dept of Medicine, Division of Allergy, Pulmonary and Critical Care Medicine, University of Wisconsin School of Medicine and Public Health, Madison, WI, USA.

Correspondence: William W. Busse, University of Wisconsin Hospitals and Clinics, 600 Highland Avenue, Madison, WI 53792, USA. E-mail: wwbRmedicine.wisc.edu

@ERSpublications

Response to omalizumab is not dependent on peripheral blood eosinophil values http://ow.ly/J7KT30jOlYC

Cite this article as: Busse WW. Are peripheral blood eosinophil counts a guideline for omalizumab treatment? STELLAIR says no! Eur Respir J 2018; 51: 1800730 [https://doi.org/10.1183/13993003.00730-2018].

Biologics for the treatment of severe asthma are assuming an ever expanding and important role to gain disease control when current therapeutics are not effective [1,2]. Although the number of asthma patients with severe disease is relatively small, $10-15 \%$, when compared to the overall prevalence of asthma, the burden of disease in this group is large from many perspectives including compromises to lifestyle, risks for recurrent and severe exacerbations, hospitalisations, unscheduled care needs, potential for adverse effects from medications, and medical costs. Therefore, severe asthma carries a dominant disease burden for the affected patient $[3,4]$.

In most countries, four biologics are currently available for severe asthma care: omalizumab, mepolizumab, reslizumab and benralizumab. In addition, dupilumab is under review for approval. These biologics target key pathways in the pathophysiology of asthma: IgE, interleukin (IL)-5 and IL-4/IL-3. The results from traditional clinical protocols, real-life trials and post-marketing surveys provide evidence of the effectiveness of biologics in appropriately defined patient populations. As all of the currently approved biologics modify Type (T)2 inflammation, the targeted patient profiles in which these biologics will be used have similarities. Consequently, a major question for the clinician is what are the most effective biomarkers currently available to guide decisions on the selection of a biologic and who is the patient with severe asthma most likely to benefit. It is the process of lining up the "right biologic to the right patient".

In this issue of the European Respiratory Journal, HumBERT et al. [5] in France have addressed this question by evaluating the value of peripheral blood eosinophil counts as a biomarker to predict the likelihood of a beneficial response to omalizumab, a monoclonal antibody directed against IgE. Up to this point, the available directive information on this relationship has been varied and confusing.

The currently approved biologics in asthma are designed to inhibit selective components of the T2 inflammatory pathways in asthma: IgE (omalizumab) and eosinophils (mepolizumab, reslizumab and benralizumab). To guide the selection of patients with evidence of T2 inflammation in their disease, a number of biomarkers have been selected: IgE, eosinophils (peripheral blood and/or sputum), exhaled nitric oxide ( FeNO) and periostin. The actions of mepolizumab, reslizumab and benralizumab are directed against the IL-5 pathway [6-8]. They achieve their effect by binding to IL-5 (mepolizumab and reslizumab) or the IL-5 receptor (benralizumab) on cells. Collectively, the target for anti-IL-5 treatment is 
the eosinophil and benefit from their use in severe asthma is seen, primarily, when peripheral blood eosinophils are increased, e.g. $\geqslant 150$ cells $\mu \mathrm{L}^{-1}$. Clinical efficacy with an anti-IL-5 is not predictable when eosinophil values are low (i.e. $<150$ cells $\mu \mathrm{L}^{-1}$ ) [6]. Consequently, and effectively, the prescribing of anti-IL-5 biologics is directed toward "eosinophilic" asthma.

The predictive process with omalizumab is not as straightforward as being an eosinophil-directed treatment, even though the T2 pathway is involved. Rather, the selection of an omalizumab candidate begins with evidence for allergic asthma, usually defined as evidence of $\operatorname{IgE}$ sensitisation to environmental allergens [9, 10]. Dosing with omalizumab is based on serum concentrations of IgE. However, as a rule, IgE parameters have not proven to be valuable biomarkers in predicting a beneficial response to omalizumab.

The aim of STELLAIR (Next Steps Toward personalised care: EvaLuating responders to XoLAIR treatment in patients with severe allergic asthma) was to determine and describe the effectiveness of omalizumab in relation to peripheral blood values which were determined in the 12 months prior to initiation of anti-IgE [5]. There are many unique and clinically relevant findings emerging from STELLAIR. Their findings provide information to help direct and substantiate whether there should be limits to selecting candidates for omalizumab based on eosinophil counts as a predictive biomarker to more effectively identify "responders" to anti-IgE treatment.

To appreciate the findings from STELLAIR, it is helpful to review the approach used by HuMBERT et al. [5] to address this question and to then put their findings in appropriate perspective. First, STELLAIR was a noninterventional, retrospective, observational study conducted in one country, France. Second, study participation included an invitation to 510 physicians (hospital-based or paediatric pulmonologists), of whom 102 accepted with 80 sites eventually participating. Third, this was a real-world study, and 879 participants met the eligibility criteria and were recruited. The inclusion criteria were straightforward and directly relevant to clinical practice: any patient $\geqslant 6$ years of age who had been treated with omalizumab for severe allergic asthma (SAA); had a blood eosinophil determination within the 12 months prior to initiation of omalizumab; had the number of asthma exacerbations recorded in the year prior to omalizumab treatment; had a documented physician evaluation of the response to omalizumab 46 months after beginning treatment; and had the number of exacerbations recorded during treatment with omalizumab. The premise of the investigators was to replicate an approach that would parallel and conform to standards of practice in France.

The investigators defined participants as a "responder" to omalizumab by one of three approaches and criteria. 1) The physician's overall evaluation by GETE (Global Evaluation of Treatment Effectiveness), which is a five-point scale with excellent (complete control of asthma) or good (marked improvement) designating a responder [11]. GETE is a subjective assessment, but has proven to be predictive of a beneficial response to omalizumab when assessed 16 weeks into treatment. 2) A decrease in the annual exacerbation rate of at least $40 \%$ after $4-6$ months of omalizumab treatment. 3) A combination of the GETE evaluation and a $40 \%$ reduction in the annual exacerbation rate. From my perspective, these are reasonable outcomes to determine and designate a "responder," especially the annualised $40 \%$ reduction in exacerbation values.

The participants enrolled in this open trial had well substantiated evidence of SAA (table 1 in [5]). Both minors $(n=149)$ and adults $(n=723)$ used large doses of inhaled corticosteroids (ICS) with a mean daily $\left(\mu \mathrm{g} \cdot \mathrm{day}^{-1}\right.$ ) dose of beclomethasone equivalency of $1545 \mu \mathrm{g} \cdot \mathrm{day}^{-1}$ and $1990 \mu \mathrm{g} \cdot \mathrm{day}^{-1}$ respectively. Moreover, $20.4 \%$ of the adults were taking an average daily prednisone dose of $20.4 \mathrm{mg}$. In the preceding year, the minors had a mean of 5.2 exacerbations and the adults 4.3. Median peripheral blood eosinophil values were 619 cells $\mu \mathrm{L}^{-1}$ in minors and 308 cells $\mu \mathrm{L}^{-1}$ in adults. The occurrence of frequent exacerbations of asthma despite treatment with large doses of ICS reflects uncontrolled disease.

During the treatment observation period, participating physicians provided care, modified patient's medications, assessed comorbidities and exacerbations, and evaluated the response to omalizumab using the methods noted above. Assessing a "responder" by annualised reductions in exacerbations found a mean decrease of $78.5 \%$ in minors and $71.1 \%$ in adults. These values generally exceed observations made in most previous randomised, double-blind placebo-controlled trials. To ascertain the relationship of peripheral blood eosinophils to rates of response to omalizumab, the investigators found reductions in exacerbations were similar in participants over a wide range of blood eosinophil counts, < or $\geqslant 150$ cells $\mu \mathrm{L}^{-1}$ or $<$ or $\geqslant 300$ cells $\mu \mathrm{L}^{-1}$ (figure $3 \mathrm{~b}$ in [5]) as well as across all gradients from $<150$ to $\geqslant 1000$ cells $\mu \mathrm{L}^{-1}$ (figure 4 in [5]).

How do these results compare to previous observations and how can observed differences from STELLAIR be understood? In the EXTRA study, for example, HANANIA et al. [12] evaluated omalizumab treatment in adolescent and adult subjects with severe asthma (high-dose combination ICS/long-acting $\beta_{2}$-agonist 
(LABA) and oral corticosteroids in some subjects). Omalizumab, compared with placebo, reduced exacerbation rates by $25 \%$. The investigators also explored the prevention of exacerbations with omalizumab in relationship with peripheral blood eosinophil values and FeNO. In the EXTRA study, the cut-off values for these biomarkers were: eosinophils $\leqslant 260$ cells $\mu \mathrm{L}^{-1}$ versus $>260$ cells $\mu \mathrm{L}^{-1}$, and FeNO $<19.5 \mathrm{ppb}$ versus $\geqslant 19.5 \mathrm{ppb}$ [13]. The prevention of exacerbations by omalizumab was greater in the presence of the higher eosinophil values, a 32\% versus $9 \%$ reduction. However, as pointed out in the STELLAIR discussion, these differential effects may relate to fewer exacerbations in the placebo group who had low eosinophils at baseline, and, as a consequence, may not have been a good test to determine responsiveness in relationship to blood eosinophil values in this particular study.

We reported on 328 uncontrolled asthma patients who were randomised to omalizumab versus placebo for 24 weeks of treatment [14]. Overall, there was no difference in the frequency of exacerbation rates between treatment with omalizumab ( 0.21 per 24 weeks) versus placebo ( 0.26 per 24 weeks). The two subject groups were divided by entry levels of eosinophil values: $<300$ cells $\mu \mathrm{L}^{-1}$ versus $\geqslant 300$ cells $\mu \mathrm{L}^{-1}$. In this study, exacerbation rates in the high eosinophil group on placebo were 0.59 per 24 weeks and 0.16 per 24 weeks in the low eosinophil group. Only in the high eosinophil group did omalizumab significantly reduce exacerbations versus placebo $(0.25$ versus $0.59, \mathrm{p}=0.0125)$. Because the primary outcome was not achieved in the overall study, the differential effect of omalizumab in relation to blood eosinophils at entry is difficult to ascertain.

CASAlE et al. [15], in contrast, found a greater response in patients treated with omalizumab when peripheral blood eosinophils were $\geqslant 300$ cells $\mu \mathrm{L}^{-1}$. This analysis consisted of pooled data from two, pivotal, phase III trials which were placebo-controlled, and represented a total of 1071 subjects. The relationship of eosinophil levels to response to omalizumab was based on comparison of exacerbation rates. In their analysis, exacerbation rates increased in relation to peripheral blood eosinophils as assessed in placebo-treated subjects. In subjects with eosinophil values $\geqslant 300 \mathrm{cells} \cdot \mu \mathrm{L}^{-1}$, omalizumab reduced exacerbations by $67 \%$; there was a $45 \%$ reduction in exacerbations with omalizumab in subjects with values $<300$ cells $\mu \mathrm{L}^{-1}$. However, all parameters of greater disease severity, history of emergency asthma treatment, hospitalisation, forced expiratory volume in $1 \mathrm{~s} \%$ predicted, ICS dose and LABA use, were associated with a greater reduction in exacerbation by omalizumab, suggesting the preventive benefit may have been more related to disease severity, as noted by others [16]. Furthermore, the majority of assessed participants (914 out of 1071) did not use LABA, as these studies were carried out prior to a more frequent use of combination therapy in severe disease.

In the PROSE study [17], observations were made on the relationship of blood eosinophils to omalizumab effects on asthma exacerbations. The study design was very different from STELLAIR. First, the participants were children and adolescents (6-17 years of age). Second, there was an extended run-in phase (4-9 months) to achieve asthma control by adjustment of treatment medications. Third, following the run-in, the school-age participants were given either omalizumab, a boost in ICS, or maintained on guideline care for the next 4 months as they returned to school. During the run-in, 141 subjects had an exacerbation; of this group, 105 were subsequently randomised to omalizumab (plus guideline care) and 24 to guideline treatment alone. Omalizumab treatment reduced exacerbations by $88 \%$ in the group who had had an exacerbation in the run-in, whereas in the group that did not experience an exacerbation during the run-in, exacerbation rates in both the placebo (13.6\%) and omalizumab (12.2\%) groups were low and similar. In the group who had an exacerbation during the run-in, the mean group eosinophil count was 350 cells $\mu \mathrm{L}^{-1}$ versus 280 cells $\mu \mathrm{L}^{-1}$ in the non-exacerbation group $(\mathrm{p}<0.01)$. Omalizumab-associated prevention of exacerbations was greater in the presence of higher eosinophil values. Did this mean that higher eosinophil values predicted a response to omalizumab or that this group had a greater risk for exacerbation and hence the potential to see an effect from omalizumab? This is not clearly established. Moreover, do these findings in PROSE contradict the results in STELLAIR? From my perspective, no, as the study design, patient populations and number of treated subjects was quite different.

Although obvious shortcomings exist with any open label study, there is definite merit and clinical benefit to be gained from STELLAIR. Their data suggest that eosinophils, as a biomarker, do not predict the likelihood for a beneficial response to omalizumab. Clinically, this is informative as it may provide support for use of omalizumab in "non-T2" or "low T2" patient groups with SAA and achieve a response. Allergic asthma has a pathogenic basis for $\mathrm{T} 2$ inflammation, i.e. allergic sensitisation and elevated IgE. Although not the goal or purpose of STELLAIR, these findings suggest that presumably accepted T2 inflammation is not fully encompassed by eosinophils alone. This is not surprising as severe asthma is complicated, complex and heterogeneous. More biomarkers, and particularly more informative biomarkers, are needed to precisely define populations with severe asthma. It is also important to define the pathways by which, and through which, omalizumab and other biologics act. STELLAIR, however, has begun to provide 
guidance for a more expanded use of omalizumab when evaluated by peripheral eosinophil counts, with evidence that response occurs over a large eosinophil range.

Conflict of interest: W.W. Busse reports personal fees (consultancy) from 3M, Boehringer Ingelheim, AstraZeneca, GlaxoSmithKline, Novartis, Sanofi-Regeneron and Teva; personal fees (data safety monitoring board) from Boston Scientific and Genentech; personal fees (royalties) from Elsevier; personal fees (educational videos) from Medscape; and personal fees ( joint oversight committee) from ICON, all outside the submitted work.

\section{References}

1 Katial RK, Bensch GW, Busse WW, et al. Changing paradigms in the treatment of severe asthma: the role of biologic therapies. J Allergy Clin Immunol Pract 2017; 5: S1-S14.

2 Israel E, Reddel HK. Severe and difficult-to-treat asthma in adults. N Engl J Med 2017; 377: 965-976.

3 O'Neill S, Sweeney J, Patterson CC, et al. The cost of treating severe refractory asthma in the UK: an economic analysis from the British Thoracic Society Difficult Asthma Registry. Thorax 2015; 70: 376-378.

4 Chung KF, Wenzel SE, Brozek JL, et al. International ERS/ATS guidelines on definition, evaluation and treatment of severe asthma. Eur Respir J 2014; 43: 343-373.

5 Humbert M, Taillé C, Mala L, et al. Omalizumab effectiveness in patients with severe allergic asthma according to blood eosinophil count: the STELLAIR study. Eur Respir J 2018; 51: 1702523.

6 Ortega HG, Liu MC, Pavord ID, et al. Mepolizumab treatment in patients with severe eosinophilic asthma. $N$ Engl J Med 2014; 371: 1198-1207.

7 FitzGerald JM, Bleecker ER, Nair P, et al. Benralizumab, an anti-interleukin-5 receptor alpha monoclonal antibody, as add-on treatment for patients with severe, uncontrolled, eosinophilic asthma (CALIMA): a randomised, double-blind, placebo-controlled phase 3 trial. Lancet 2016; 388: 2128-2141.

8 Castro M, Zangrilli J, Wechsler ME, et al. Reslizumab for inadequately controlled asthma with elevated blood eosinophil counts: results from two multicentre, parallel, double-blind, randomised, placebo-controlled, phase 3 trials. Lancet Respir Med 2015; 3: 355-366.

9 Busse W, Corren J, Lanier BQ, et al. Omalizumab, anti-IgE recombinant humanized monoclonal antibody, for the treatment of severe allergic asthma. J Allergy Clin Immunol 2001; 108: 184-190.

10 Humbert M, Busse W, Hanania NA, et al. Omalizumab in asthma: an update on recent developments. J Allergy Clin Immunol Pract 2014; 2: 525-536.

11 Bousquet J, Rao S, Manga V. Global evaluation of treatment effectiveness (GETE) is an accurate predictor of response to omalizumab in patients with severe allergic asthma: A pooled analysis. Eur Respir J 2014; 44: Suppl. 58, P3483.

12 Hanania NA, Alpan O, Hamilos DL, et al. Omalizumab in severe allergic asthma inadequately controlled with standard therapy: a randomized trial. Ann Intern Med 2011; 154: 573-582.

13 Hanania NA, Wenzel S, Rosen $\mathrm{K}$, et al. Exploring the effects of omalizumab in allergic asthma: an analysis of biomarkers in the EXTRA study. Am J Respir Crit Care Med 2013; 187: 804-811.

14 Busse W, Spector S, Rosen K, et al. High eosinophil count: a potential biomarker for assessing successful omalizumab treatment effects. J Allergy Clin Immunol 2013; 132: 485-486.

15 Casale $\mathrm{TB}$, Chipps $\mathrm{BE}$, Rosen $\mathrm{K}$, et al. Response to omalizumab using patient enrichment criteria from trials of novel biologics in asthma. Allergy 2018; 73: 490-497.

16 Bousquet J, Wenzel S, Holgate S, et al. Predicting response to omalizumab, an anti-IgE antibody, in patients with allergic asthma. Chest 2004; 125: 1378-1386.

17 Teach SJ, Gill MA, Togias A, et al. Preseasonal treatment with either omalizumab or an inhaled corticosteroid boost to prevent fall asthma exacerbations. J Allergy Clin Immunol 2015; 136: 1476-1485. 